RECYT

Año 21 / № 32 / 2019 / 64-70

\title{
Optimización de la hidrólisis enzimática de la cascarilla de arroz
}

\section{Optimisation of the enzymatic hydrolysis of rice husk}

\author{
Ana María Arismendy $y^{1, *}$, Andrés Felipe Villa Restrepo ${ }^{2}$, \\ Wilman Alcaraz Zapata ${ }^{2}$, Ester R. Chamorro ${ }^{1}$, María C. Area ${ }^{3}$ \\ 1- (UTN-CONICET), French 414, Resistencia, Argentina. \\ 2- Grupo de Biotransformación, Escuela de Microbiología, Universidad de Antioquia, Calle 67 N 53-108, Medellín, Colombia. \\ 3- Programa de Celulosa y Papel, Instituto de Materiales de Misiones, IMAM (UNaM-CONICET), Félix de Azara 1552, \\ Misiones, Argentina. \\ *E-mail: anaarismendy25@gmail.com
}

\section{Resumen}

El objetivo de este trabajo fue encontrar las condiciones óptimas de hidrólisis enzimática de la cascarilla de arroz pretratada con hidróxido de sodio en autoclave a $121^{\circ} \mathrm{C}$. Se evaluaron las variables: FPU/g sustrato, pH, agitación, tiempo, temperatura y concentración de surfactante mediante un diseño de cribado, resultando estadísticamente significativas todas las variables. Seguidamente se aplicó un diseño de optimización para las variables: pH, FPU y tiempo, descartando las de menor nivel de significación. Finalmente, las mejores condiciones encontradas en los diseños anteriores fueron ( $\mathrm{pH}$ 5.0, 13 h, y $30 \mathrm{FPU} / \mathrm{g}$ de sustrato) posteriormente se aplicaron estas condiciones en experiencias para evaluar la influencia del aumento de celulosa hidrolizable, usando $6 \% ; 8 \%$ y $10 \%$ de celulosa disponible con rendimientos de sacarificación de $74 \%$, $42 \%$ y $16 \%$.

Palabras clave: Cascarilla de arroz; Hidrólisis enzimática; Biomasa Lignocelulósica; Condiciones óptimas; Bioetanol.

\section{Abstract}

The objective of this work was to find the optimal conditions of enzymatic hydrolysis of the rice husk pretreated with sodium hydroxide in an autoclave at $121^{\circ} \mathrm{C}$. Screening design showed that all the selected variables (FPU / $\mathrm{g}$ substrate, $\mathrm{pH}$, agitation, time, temperature and concentration of surfactant) were statistically significant. Then an optimization design was applied for the variables: pH, FPU and time, discarding the ones with the lowest level of significance. Finally, the best conditions found in the previous designs were (pH 5.0, $13 \mathrm{~h}$, and $30 \mathrm{FPU} / \mathrm{g}$ of substrate) subsequently these conditions were applied in experiences to evaluate the influence of the increase in hydrolysable cellulose, using $6 \% ; 8 \%$ and $10 \%$ of cellulose available giving saccharification yields of $74 \%, 42 \%$ and $16 \%$.

Keywords: Rice husk; Enzymatic hydrolysis; Lignocellulosic Biomass; Optimal Conditions; Bioethanol.

\section{Introducción}

Debido al agotamiento de los combustibles fósiles renovables y la problemática ambiental, se buscan nuevas alternativas para la producción de biocombustibles. En los últimos años se han producido grandes cantidades de bioetanol a partir de caña de azúcar y maíz, pero esto ha generado problemáticas a nivel social, económico y político, porque se compite por el sector alimenticio, debido a que es necesario cultivar grandes cantidades para abastecer la producción de bioetanol y el sector alimenticio [1]. Una alternativa son los biocombustibles de segunda generación provenientes de desechos agrícolas o biomasa lignocelulósica. La FAO afirmó que para el año 2018/ 2019 se producirían cerca de 510,6 millones de toneladas de arroz, la mayor parte destinada a cubrir las necesidades alimentarias. En Sudámerica se produjeron 24 millones de toneladas de arroz, aun así, países como Brasil, Bolivia, Colombia, Ecuador y Argentina sufrieron una reducción de la producción por condiciones climáticas no favorables, y recorte de remuneraciones [2]. La biomasa generada como residuo de esta producción agrícola (paja, cáscaras) es eliminada por prácticas como la combustión [3], con impactos negativos a nivel ambiental, por lo que se ha buscado un uso a este tipo de materias primas como fuente de biocombustibles para producir bioetanol [4] .

La biomasa lignocelulósica se encuentra formada principalmente por celulosa, hemicelulosas y lignina. La celulosa es un homopolímero de largas cadenas de glucosa, las hemicelulosas son heteropolímeros de azúcares de cinco y seis átomos de carbono mientras que la lignina es un polímero aromático complejo [5]. 
Para la producción de bioetanol de biomasa lignocelulósica es necesario realizar tres procesos que comprenden pretratamiento, sacarificación enzimática y finalmente fermentación de azúcares generados en la etapa anterior [6]. El pretratamiento permite extraer la lignina y hemicelulosas. Debe cumplir otras características como aplicabilidad a diversos sustratos, bajo costo y consumo energético [7].

El pretratamiento debe contribuir a mejorar la formación de azúcares en la hidrólisis y no generar inhibidores que perjudiquen la eficiencia de la hidrólisis y fermentación [1]. Factores como el área superficial, la cristalinidad de la celulosa y el contenido de lignina del material dificultan la eficiencia de la hidrólisis, por lo que es necesario aplicar un pretratamiento que facilite la actividad de las enzimas [8].

Entre los pretratamientos reportados por la literatura, se encuentra el alcalino que rompe los enlaces entre lignina, hemicelulosas y celulosa, ayudando así a la eliminación de lignina y hemicelulosa $[9,10]$. En este proceso se hincha la biomasa, y dependiendo de la temperatura predominan las reacciones de peeling (temperaturas elevadas) o de deacetilación, consistentes en la pérdida de acetilos de los xilanos y glucomananos (a temperaturas moderadas). Este pretratamiento es más efectivo sobre latifoliadas y gramíneas que sobre coníferas [11].

La hidrólisis enzimática genera la ruptura de polímeros de celulosa y hemicelulosas, para obtener monómeros como glucosa y xilosa [12]. La hidrólisis enzimática es un proceso catalítico donde participan diferentes enzimas que actúan sinérgicamente. Las endoglucanasas se encargan de hidrolizar los enlaces $\beta-(1,4)$ glucosídicos para generar extremos de cadena no reductores; las celobiohidrolasas actúan sobre los extremos no reductores y reductores hidrolizando cadenas cortas de celulosa, liberando celobiosa y las $\beta$-glucosidasas hidrolizan la celobiosa y oligosacáridos pequeños a glucosa [13]

Las enzimas utilizadas para la sacarificación son celulasas las cuales poseen especificidad por los sustratos que poseen celulosa [14].

Este trabajo buscó optimizar las condiciones de hidrólisis enzimática de la cascarilla de arroz después de ser pretratada con hidróxido de sodio a temperatura moderada.

\section{Materiales y Métodos}

\section{Materias primas e insumos}

La cascarilla de arroz fue provista por la Central Mayorista de la ciudad de Medellín, Colombia. La cascarilla inicialmente fue sometida a un tratamiento mecánico en molino para disminuir el tamaño de partícula. Se utilizaron enzimas donadas por Cellic ${ }^{\circledR}$ CTec2 Novozymes Latin America Araucaría Brasil.

\section{Caracterización de la cascarilla de arroz}

Se caracterizó la cascarilla sin pretratamiento y pretratada utilizando las normas NREL (National Renewable Energy Laboratory). Se determinó contenido de humedad y sustancias volátiles (NREL/TP-510-4262) [15], contenido de sustancias extractivas solubles en agua y etanol (NREL/ TP 510-42621) [16], carbohidratos y lignina (NREL/TP510-42618) [17], y de cenizas (NREL/TP-510-42622) [18].

\section{Pretratamiento de la materia prima}

Se pretrató la cascarilla de arroz con hidróxido de sodio $3 \% \mathrm{p} / \mathrm{V}$ y $10 \% \mathrm{p} / \mathrm{V}$ de biomasa durante una hora a $121^{\circ} \mathrm{C}$ en autoclave según protocolo optimizado por López, 2012 [19].

\section{Hidrólisis enzimática}

Se realizó mediante protocolo propuesto en la norma NREL para hidrólisis enzimática (NREL/TP-510-42629) [20] modificando la cantidad de carga enzimática que se utiliza en este trabajo con $30 \mathrm{FPU}$ FPU/g sustrato y la utilización del surfactante Tween 80 para mejorar el funcionamiento de la hidrólisis enzimática y además, no se agregaron antibióticos. Se determinó la humedad y sustancias volátiles (NREL/TP-510-42621) [15] de la cascarilla pretratada. Se utilizó un volumen de trabajo de $200 \mathrm{~mL}$ en todos los diseños aplicados, incluyendo los ensayos preliminares para aumento de celulosa hidrolizable. El proceso de hidrólisis enzimática se aplicó a la cascarilla pretratada según dos diseños experimentales y se realizó un ensayo preliminar para verificar un eventual aumento de celulosa hidrolizable.

Las condiciones de los diseños pueden observarse en la Tabla 1.

Tabla 1: Condiciones de trabajo en los diseños experimentales aplicados con $1 \%$ de celulosa hidrolizable

\begin{tabular}{|c|c|c|c|c|c|c|}
\hline $\begin{array}{l}\text { Tipo de } \\
\text { diseño }\end{array}$ & $\begin{array}{c}\mathrm{FPU} / \mathrm{g} \\
\text { sustrato }\end{array}$ & $\mathrm{pH}$ & $\begin{array}{c}\text { Temperatura } \\
\left({ }^{\circ} \mathrm{C}\right)\end{array}$ & $\begin{array}{c}\text { Agitación } \\
\text { (rpm) }\end{array}$ & $\begin{array}{c}\text { Tiempo } \\
\text { (h) }\end{array}$ & $\begin{array}{c}\text { Concentración } \\
\text { de surfactante } \\
(\mathrm{g} / \mathrm{L})\end{array}$ \\
\hline \multirow{2}{*}{ Cribado } & 10 & 4,8 & 32 & 100 & 8 & 0,1 \\
\hline & 20 & 6,0 & 37 & 180 & 12 & 0,3 \\
\hline \multirow{5}{*}{ Optimización } & 3 & 4,1 & \multirow{5}{*}{37} & \multirow{5}{*}{180} & 6 & \multirow{5}{*}{0,3} \\
\hline & 10 & 4,5 & & & 8 & \\
\hline & 25 & 5,2 & & & 13 & \\
\hline & 40 & 6,0 & & & 18 & \\
\hline & 47 & 6,3 & & & 20 & \\
\hline
\end{tabular}

El volumen de trabajo del hidrolizado que corresponde a $200 \mathrm{~mL}$ se completó con el buffer citrato de sodio correspondiente a $100 \mathrm{~mL}, 98 \mathrm{~mL}$ aproximadamente de agua destilada, las cantidades de enzima, de surfactante Tween 80 (g/L) y ajuste de $\mathrm{pH}$, se especifican en la Tabla 
1, según corresponde a cada diseño realizado. A partir de los resultados obtenidos del diseño de cribado se dejaron fijas las variables: concentración de surfactante, velocidad de agitación y temperatura.

Para desarrollar a futuro fermentaciones e hidrólisis y fermentación simultánea con $S$. cerevisiae, los rangos de cada una de las variables se fijaron según bibliografía consultada y observando que a $37^{\circ} \mathrm{C}$ se generan rendimientos aceptables de azúcares fermentables y también es la temperatura máxima que podrían llegar a soportar microorganismos como $S$. cerevisiae, al igual que el resto de condiciones optimizadas en los procesos realizados en este trabajo, que también son favorables para los procesos posteriores. Seguidamente las variables que se estudiaron en el diseño de optimización fueron $\mathrm{pH}$, tiempo y FPU/ g sustrato. Del diseño de optimización se obtuvieron las condiciones en las que se podrían llegar a obtener rendimientos cercanos al 70\% de azúcares, pero con una cantidad muy significativa de enzima. Por esta razón se realizaron ensayos con una cantidad menor de enzima, tomada como referencia de la gráfica de contornos, para llegar a obtener rendimientos de azúcares de hasta un 52\%.

La concentración y el rendimiento de los azúcares en la hidrólisis enzimática se obtuvieron como se muestra en las ecuaciones 1 y 2 .

Concentración de azúcares $=(\mathrm{g}$ cascarilla $\operatorname{secos} * 0,51 / 0,9)=$ $\mathrm{g} / \mathrm{L}$ azúcares

\% Rendimiento de azúcares= $(\mathrm{g}$ azúcares obtenidos $/ \mathrm{g}$ azúcares teóricos $* 100)=\%$ azúcares

Donde: 0,51 es la cantidad de celulosa en el material que fue previamente determinada por HPLC y 0,9 es el factor de hidrólisis.

El rendimiento de azúcares se obtiene de los g cuantificados por DNS en los diseños de hidrólisis enzimática, y los g de azúcares teóricos son obtenidos de la cantidad de celulosa que se utiliza en cada diseño dividido entre 0,9 que es el factor de hidrólisis. Las condiciones correspondientes de cada diseño se explican en la Tabla 1.

\section{Métodos analíticos}

Los carbohidratos se determinaron por el método de azúcares reductores DNS (ácido dinitrosalicílico) [21] para los diseños realizados y cromatografía líquida en fase reversa (Waters HPLC System), usando la columna Aminex HPX87H, HPX-87P marca BIORAD para la caracterización de la materia prima original y después de pretratada.

Las actividad enzimática expresada en FPU (Filter Paper Unit), la capacidad que posee la enzima para sacarificar celulosa, se determinó siguiendo el procedimiento detallado por IUPAC (International Union of Pure and Applied Chemistry, Ghose 1987) [22].

\section{Diseños experimentales}

Para la hidrólisis enzimática, con la finalidad de encontrar los factores significativos, se aplicó un diseño de cribado de un octavo de fracción, 4 puntos centrales y resolución 3, por duplicado, completando una totalidad de 24 experiencias.

Para obtener las condiciones con mayores rendimientos de producción de azúcares se propuso un diseño de optimización rotable con 5 puntos centrales, por duplicado, completando 38 experiencias, se trabajó con el error puro en los diseños.

Con las condiciones óptimas de hidrólisis enzimáticas obtenidas en el diseño anterior, se realizaron ensayos de optimización/sup respuesta para evaluar el rendimiento de azúcares con 6,8 y 10\% de celulosa hidrolizable. Se realizó por duplicado totalizando 6 experiencias. Los análisis estadísticos se realizaron con el programa Startgraphics Centurión.

\section{Resultados y Discusión}

\section{Caracterización de la cascarilla de arroz}

La composición de la cascarilla de arroz sin pretratamiento (original) fue: lignina insoluble en ácido 20,4\%; extractivos totales $15 \%$; inorgánicos $10 \% ; 55 \%$ carbohidratos totales (glucano y xilano), mientras que su composición después del pretratamiento fue: lignina insoluble en ácido $11,3 \%$; inorgánicos 2,0\%; 51\% glucano; $13 \%$ de extractivos totales y $16,1 \%$ xilano. La caracterización de la biomasa después del pretratamiento se realizó para establecer la cantidad de celulosa disponible a hidrolizar en los diseños desarrollados.

\section{Selección de variables}

Los resultados del diseño de cribado para verificar la significación de las variables sobre la hidrólisis enzimática se muestran en la Tabla 2. 
Tabla 2: Resultados del diseño de cribado de hidrólisis enzimática

\begin{tabular}{|c|c|c|c|c|c|c|}
\hline $\begin{array}{c}\text { FPU/ g } \\
\text { sustrato }\end{array}$ & $\mathbf{p H}$ & $\begin{array}{c}\text { Agitación } \\
(\mathbf{r p m})\end{array}$ & $\begin{array}{c}\text { Temperatura } \\
\left({ }^{\circ} \mathbf{C}\right)\end{array}$ & $\begin{array}{c}\text { Tiempo } \\
(\mathbf{h})\end{array}$ & $\begin{array}{c}\text { Concentración } \\
\text { surfactante(g/L) }\end{array}$ & $\begin{array}{c}\text { Rendimiento } \\
\text { azúcares (\%) } \\
(\mathbf{D} . \mathbf{S})\end{array}$ \\
\hline 10 & 4,8 & 100 & 37 & 12 & 0,3 & $41,0( \pm 0,0)$ \\
\hline 20 & 6,0 & 100 & 37 & 8 & 0,1 & $46,0( \pm 0,0)$ \\
\hline 20 & 6,0 & 180 & 37 & 12 & 0,3 & $49,0( \pm 0,0)$ \\
\hline 10 & 4,8 & 180 & 37 & 8 & 0,1 & $29,5( \pm 0,5)$ \\
\hline 20 & 4,8 & 100 & 32 & 8 & 0,3 & $48,7( \pm 0,1)$ \\
\hline 10 & 6,0 & 100 & 32 & 12 & 0,1 & $18,9( \pm 0,7)$ \\
\hline 15 & 5,4 & 140 & 34 & 10 & 0,2 & $41,5( \pm 0,7)$ \\
\hline 20 & 4,8 & 180 & 32 & 12 & 0,1 & $50,5( \pm 0,3)$ \\
\hline 10 & 6,0 & 180 & 32 & 8 & 0,3 & $21,0 *( \pm 0,0)$ \\
\hline * Promedio de 8 repeticiones del punto central & & \\
\hline
\end{tabular}

El análisis de la varianza indicó que las variables FPU/g sustrato, $\mathrm{pH}$, temperatura, tiempo y concentración de surfactante $\mathrm{g} / \mathrm{L}$ (Tween 80 ) son significativas en el proceso de hidrólisis enzimática $(\mathrm{p}<0,05)$, mientras que la agitación prácticamente no resultó significativa en el proceso $(\mathrm{p}$ cercano a 0,05). Esto significa que para los volúmenes y la concentración de sólidos utilizada la influencia de esta variable puede descartarse. Sin embargo, esto podría cambiar al aumentar la concentración de sólidos.

Los resultados de la influencia de la concentración de surfactante sobre la hidrólisis enzimática confirman que contribuye a mejorar la eficiencia de la interacción entre sustrato y enzima [23, 24,25], mejorando así la accesibilidad de la enzima al sustrato $[24,26]$. Con cantidades de carga enzimática en rangos de 10 a $20 \mathrm{FPU} / \mathrm{g}$ sustrato se obtuvieron rendimientos de alrededor del $50 \%$ en 12 horas como máximo. Se decidió fijar la temperatura en $37^{\circ} \mathrm{C}$ en base a la bibliografía, ya que se han reportado rendimientos significativos de etanol con levaduras comerciales (S. cerevisiae) a esa temperatura. En dichos trabajos se utilizaron velocidades de agitación de $500 \mathrm{rpm}$ verificando que es fundamental aumentar la velocidad de agitación al procesar una mayor carga de biomasa [27].

\section{Optimización de la hidrólisis enzimática}

Para realizar la optimización de la hidrólisis enzimática, según los resultados del diseño de cribado, todas las variables resultaron significativas, aunque la velocidad de agitación mostró encontrarse en el límite de la significación estadística $(p=0,05)$. Para realizar el próximo diseño se aplicaron las condiciones mostradas en la Tabla 2, manteniendo fijas las variables agitación en $180 \mathrm{rpm}$, la temperatura de $37^{\circ} \mathrm{C}$ y la concentración de surfactante $0,3 \mathrm{~g} / \mathrm{L}$. Los resultados se muestran en la Tabla 3. Se observa que con el aumento de la carga enzimática (FPU/g sustrato) lo máximo que puede obtenerse es $65 \%$ de rendimiento, con $\mathrm{pH}$ hasta 5,3 .
Tabla 3: Resultados del diseño de optimización de la hidrólisis enzimática

\begin{tabular}{|c|c|c|c|}
\hline pH & FPU/g sustrato & $\begin{array}{c}\text { Tiempo } \\
\text { (h) }\end{array}$ & $\begin{array}{c}\text { Rendimiento } \\
\text { azúcares (\%) (D.S) }\end{array}$ \\
\hline 4,5 & 10 & 8 & $28,5( \pm 0,5)$ \\
\hline 6,0 & 10 & 8 & $16,5( \pm 0,7)$ \\
\hline 4,5 & 40 & 8 & $51,3( \pm 1,8)$ \\
\hline 6,0 & 40 & 8 & $33,1( \pm 0,1)$ \\
\hline 4,5 & 10 & 18 & $30,2( \pm 0,1)$ \\
\hline 6,0 & 10 & 18 & $18,5( \pm 0,7)$ \\
\hline 4,5 & 40 & 18 & $56,7( \pm 0,4)$ \\
\hline 6,0 & 40 & 18 & $33,0( \pm 0,0)$ \\
\hline 4,1 & 25 & 13 & $41,5( \pm 0,7)$ \\
\hline 6,4 & 25 & 13 & $11,0( \pm 0,7)$ \\
\hline 5,3 & 3 & 13 & $15,5( \pm 0,7)$ \\
\hline 5,3 & 47 & 13 & $65,0( \pm 0,0)$ \\
\hline 5,3 & 25 & 6 & $47,0( \pm 0,0)$ \\
\hline 5,3 & 25 & 20,4 & $24,0( \pm 0,01)$ \\
\hline 5,3 & 25 & 13 & $42,4^{*}( \pm 1,07)$ \\
\hline \multicolumn{3}{|c|}{ *Promedio de 10 repeticiones del punto central } \\
\hline
\end{tabular}

La ecuación del modelo ajustado $\left(\mathrm{R}^{2}=90,5\right)$ se presenta a continuación. La ecuación se elaboró con variables transformadas.

Rendimiento de azúcares (\%) 42,4 - 8,6.pH+12,0. FPU/g sustrato - 2,2.Tiempo - 5,7.pH - 2,3.pH.FPU/g sustrato - 0,7.FPU/ g sustrato ${ }^{2}$ - 2,4.Tiempo ${ }^{2}$

La Figura 1 muestra la representación del modelo de rendimiento de hidrólisis enzimática a diferentes cargas enzimáticas y $\mathrm{pH}$ en un rango de 4,5-6,0 para un tiempo de $13 \mathrm{~h}$ (punto central).

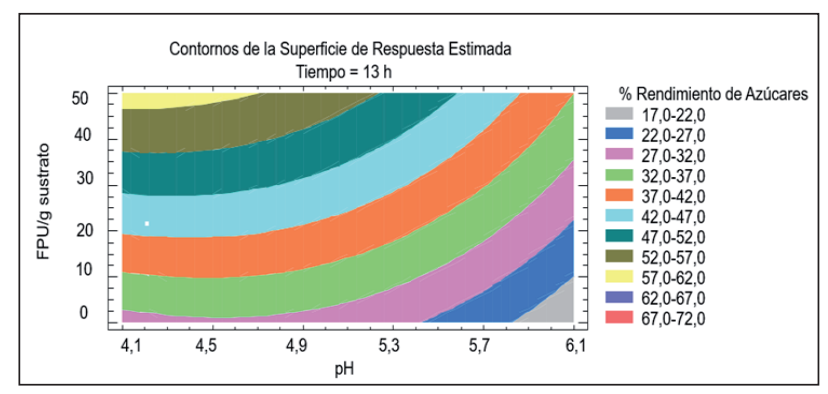

Figura 1: Gráfico de contornos de optimización del rendimiento de hidrólisis (13 horas de tratamiento)

A partir de los resultados obtenidos del diseño de optimización de la hidrólisis enzimática, se obtuvo que el máximo rendimiento de azúcares en 13 h es de aproximadamente $65 \%$ con $47 \mathrm{FPU} / \mathrm{g}$ sustrato y pH 5,0. Se ha reportado que la carga enzimática para materiales lignocelulósicos puede variar entre 7 y 33 FPU/ g sustrato, según la concentración de sustrato utilizado y tipo de material [1]; por lo que se puede deducir que la carga enzimática arrojada por el programa es demasiada alta, lo que perjudicaría la rentabilidad del proceso.

Siguiendo el modelo representado en la Figura 1, se decidió realizar ensayos de comprobación tomando como referencia los parámetros $30 \mathrm{FPU} / \mathrm{g}$ sustrato (recomendado) y $13 \mathrm{~h}$, a pH 4,5 y 5,0, considerando que en estas condiciones se obtendría un rendimiento teórico de azúcares del 52\% aproximadamente. Además, los pH 
podrían resultar favorables para los microorganismos en los procesos de hidrólisis y fermentación simultánea. Los rendimientos de azúcares (\%) para los pH de 4,5 y 5,0 fueron $44,0 \%$ y 53,0\% respectivamente. Se puede observar que la diferencia al hidrolizar biomasa a $\mathrm{pH} 4,5 \mathrm{y} \mathrm{pH}$ 5,0 es importante; siendo mayor la producción de azúcares con $\mathrm{pH} 5,0$. Por lo tanto, para el estudio siguiente se tomaron los parámetros $30 \mathrm{FPU} / \mathrm{g}$ sustrato, pH 5,0 y tiempo 13 h, variando el porcentaje de celulosa hidrolizable en 3 niveles. Los resultados obtenidos se muestran en la Tabla 4.

Tabla 4: Resultados de los ensayos de variación de celulosa hidrolizable

\begin{tabular}{|c|c|}
\hline Celulosa hidrolizable (\%) & Rendimiento de azúcares (\%) \\
\hline 6 & 75,0 \\
\hline 6 & 74,2 \\
\hline 8 & 42,1 \\
\hline 8 & 42,0 \\
\hline 10 & 16,2 \\
\hline 10 & 16,0 \\
\hline
\end{tabular}

La prueba de múltiple rangos LSD (Least significant difference), se utilizó para comparar las medias de cada uno de los porcentajes de celulosa hidrolizable, esta prueba indicó que existen diferencias significativas entre las medias de todos los niveles ensayados $(\mathrm{p}<0,05)$, resultando mejores los rendimientos obtenidos con el menor porcentaje de celulosa hidrolizable $(6,0 \%)$. Es probable que un aumento en la cantidad de celulosa genere algún tipo de inhibición por sustrato. Por otra parte, si bien la agitación casi no mostró influencia sobre el proceso al utilizar 1\% de masa, al aumentar ésta al 6\% puede que la agitación orbital no sea la más adecuada y que el sistema requiera una agitación más enérgica.

El máximo rendimiento obtenido fue de $75 \%$ en $13 \mathrm{~h}$ de tratamiento. Comparando con lo reportado en literatura, se puede afirmar que la productividad de la hidrólisis enzimática en este trabajo se encuentra en un rango aceptable teniendo en cuenta que se emplearon $13 \mathrm{~h}$ para el proceso, lo cual implica disminución de costos. La productividad de 1,7 (g/L.h) para 6\% de celulosa hidrolizable, es comparable con resultados obtenidos utilizando hojas de la planta de mandioca también pretratadas con álcali. Los autores obtuvieron una productividad de 1,4 (g/L.h) al sacarificar $6 \%$ de biomasa durante $18 \mathrm{~h}$ con una carga enzimática de $22 \mathrm{FPU} / \mathrm{g}$ sustrato [19]. En trabajos reportados utilizando cascarilla de arroz pretratada con 0,3 y $1 \%$ p/v de ácido sulfúrico con vapor a $152^{\circ} \mathrm{C}$ se obtuvieron rendimientos de hidrólisis de 55,7\% luego de 72 h de hidrólisis enzimática, aplicando $40 \mathrm{FPU} / \mathrm{g}$ sustrato usando como catalizadores las enzimas endoglucanasa (NS 50013 Product) y celobiasas (NS 50010 Product) provistas por Novozymes [28]. En un estudio realizado con lodo de Eucalyptys globulus se obtuvo una concentración de $80,4 \mathrm{~g} / \mathrm{L}$ de azúcares durante 96 h, con una carga enzimática de 25 FPU/g sustrato, y una productividad de 0,84 (g/L.h) [30] También se reporta que con pretratamiento de explosión de vapor durante 10 min a $220^{\circ} \mathrm{C}$, con una cantidad de $6,0 \%$ de celulosa hidrolizable se llego a obtener un rendimiento menor a $75 \%$ con 22FPU/g sustrato durante $96 \mathrm{~h}$ a $50^{\circ} \mathrm{C}$, utilizando las enzimas Cellic ${ }^{\circledR} \mathrm{CTec} 2$ y Cellic ${ }^{\circledR} \mathrm{HTec} 2$ [31]Otro autor reporta el uso de un proceso de deslignificación alcalina en microondas con hidrólisis posterior de 50,0 gr de cascarilla pretatada (46,0\% de celulosa), en un reactor de 3,0 litros, utilizando un coctel enzimático crudo de endoglucanasas 198,2 IU/g, xilanasas 134,3 IU/g y $\beta$-glucasidasas 147,8 IU/g (actividades de exoglucanasa 8,2 FPU/g), obteniendo rendimientos cercanos al 88\% durante 72 h [32]. En un estudio sobre rastrojo de maíz, se aplicó un diseño de superficie de respuesta para evaluar la influencia de la temperatura y la carga de hidróxido de sodio en el pretratamiento alcalino utilizando las siguientes condiciones: $130^{\circ} \mathrm{C} ; 0,70$ g de hidróxido de sodio/g de rastrojo de maíz, durante 75 minutos, obteniendo un rendimiento de azúcares de 59.8\% [33]. El resultado es comparable con el obtenido en este trabajo pero con un tiempo de $13 \mathrm{~h}$ y una carga enzimática aceptable. Esto se debe a que la cascarilla después del pretratamiento posee un porcentaje significativo de lignina y hemicelulosas que afectan la efectividad de la hidrólisis enzimática.

\section{Conclusiones}

Se evaluó la influencia de las variables FPU/g sustrato, $\mathrm{pH}$, tiempo, temperatura, concentración de surfactante $\mathrm{y}$ agitación sobre la hidrólisis enzimática de cascarilla de arroz pretratada con álcali a temperatura moderada. Todas las variables afectaron significativamente al proceso para $1 \%$ de celulosa disponible. Se observa que para el incremento de celulosa en el proceso es necesario utilizar una agitación más enérgica, para mejorar la homogenización del medio y aumentar los rendimientos de hidrólisis. Por consiguiente es viable el estudio del aumento de celulosa en el medio mejorando la agitación, ya que conlleva a obtener mayores cantidades de azúcares disponibles para la fermentación.

Utilizando 30,0 FPU/g sustrato y pH 5,0 durante $13 \mathrm{~h}$ se obtuvo un rendimiento de hidrólisis enzimática cercano al 53\%. Incrementando el porcentaje de celulosa hidrolizable al 6\%, el rendimiento de hidrólisis aumento, llegando al $75 \%$. Este valor es más que aceptable para un tiempo tan reducido (13h, en comparación con los usuales de 48-72 h).

\section{Agradecimientos}

Se agradece al CONICET (Consejo de Investigaciones Científicas y Técnicas de Argentina) por la beca de posgrado otorgada a Ana María Arismendy Pabón y también al Dr. Mario Arias Zabala, docente de la Universidad Nacional Sede Medellín, Colombia y director del grupo 
Bioconversiones, por facilitar el equipamiento para realizar algunas experiencias de este trabajo.

\section{Referencias}

1. Sun, Y y cheng J. Hydrolysis of lignocellulosic materials for ethanol production: A review, Bioresour. Technol. pp. 1-11. 2002.

2. FAO. Seguimiento del mercado del Arroz de la FAO Abril de 2017, 2017. Available at http://www.fao.org/3/ I9243ES/i9243es.pdf .

3. Singh, A; Tuteja S; Singh, N; Bishnoi, N. R. Enhanced saccharification of rice straw and hull by microwave-alkali pretreatment and lignocellulolytic enzyme production, Bioresour. Technol. pp. 1773-1782. 2011.

4. Moniruzzaman, M. Effect of steam explosion on the physicochemical properties and enzymatic saccharification of rice straw, Appl. Biochem. Biotechnol. - Part A Enzym. Eng. Biotechnol. pp. 283-297. 1996.

5. Rubin, E. M. "Genomics of cellulosic biofuels, Nature. pp. 841-845. 2008.

6. B. C. Saha and M. A. Cotta. Enzymatic saccharification and fermentation of alkaline peroxide pretreated rice hulls to ethanol, vol. 41, pp. 528-532, 2007.

7. Alvira, P; Tomás-Pejó, E; Ballesteros, M; Negro, M. J. Pretreatment technologies for an efficient bioethanol production process based on enzymatic hydrolysis: A review, Bioresour. Technol. pp. 4851-4861.2010.

8. Lynd, L.R; Weimer, P.J; van Zyl, W.H; Pretorius I.S. Microbial Cellulose Utilization: Fundamentals and Biotechnology. pp. 508. 2004.

9. Gáspár, M; Kálmán, G; Réczey, K. Corn fiber as a raw material for hemicellulose and ethanol production. Process Biochem. pp. 1135-1139. 2007.

10. Hahn-Hägerdal, B; Galbe, M; Gorwa-Grauslund, M. F; Lidén, G; Zacchi, G. Bio-ethanol - the fuel of tomorrow from the residues of today. Trends Biotechnol. 2006.

11. Hon, D.N.S. Chemical Modification of Lignocellulosic Materials, Editor. CRC Press Taylor y Francis Group. London. 1996.

12. Taherzadeh, м. J у Karimi к. Enzyme-based Hydrolysis Processes for Ethanol from Lignocellulosic Materials: A Review, vol. 2, no. 4. 2007.

13. Wood, T. M and Mccrae, s. Synergism Between Enzymes Involved in the Solubilization of Native Cellulose. Hydrolys. Cellul. Mech. Enzym. Acid Catal. vol. 181, no. 1. 1979 .

14. т. K. Ghose. International Union of Pure Commission on Biotechnology, Measurement of Cellulasae Activities. Pure Appl. Chem., vol. 59, no. 2, pp. 257-268, 1987.

15. Sluiter, A. y col. Determination of total solids in biomass and total dissolved solids in liquid process samples. Natl. Renew. Energy Lab. pp. 3-5. 2008.

16. Sluiter, A. y col. Determination of Extractives in Biomass.
Natl. Renew. Energy Lab. 2008.

17. Sluiter, A. y col. Determination of structural carbohydrates and lignin in Biomass, Natl. Renew. Energy Lab. p. 17. 2004.

18. Sluiter, A y col. Determination of Ash in Biomass. Natl. Renew. Energy Lab. pp. 1-8. 2005.

19. López,N.L. Produccion de Etanol a Partir de Residuos Lignocelulosicos de Yuca (Manihot esculenta Crantz) Mediante SHF y SSF en Modo Batch y Fed Batch, 2012.

20. Resch, M. G.; Baker, J. O; Decker, S. R. Low Solids Enzymatic Saccharification of Lignocellulosic Biomass. Natl. Renew. Energy Lab.pp. 1-9, 2015.

21. [21] G. L. Miller. Use of Dinitrosalicylic Acid Reagent for Determination of Reducing Sugar Analytical Chemistry. Vol. 31, No 3, p.426-428, March 1959.

22. Ghose, т. K. International Union of Pure Commission on Biotechnology, Measurement of Cellulasae Activities. Pure Appl. Chem. pp. 257-268. 1987.

23. Eriksson, T; Börjesson, J; Tjerneld, F. Mechanism of surfactant effect in enzymatic hydrolysis of lignocellulose, Enzyme Microb. Technol. pp. 353-364. 2002.

24. Kaar, w. E. y Holtzapple, M. т. Benefits from Tween during enzymic hydrolysis of corn stover. Biotechnol. pp. 419-427. 1998.

25. Malmsten, M y Van Alstine, J. M. Adsorption of poly(ethylene glycol) amphiphiles to form coatings which inhibit protein adsorption, J. Colloid Interface Sci. pp. 502-512, 1996.

26. Helle, S. S; Duff, S. J. B; Cooper, D. G. Effect of surfactants on cellulose hydrolysis. Biotechnol. Bioeng .pp. 611617.1993.

27. Castaño, H. M; Cardona ,B. C; Gomez, M; Cárdenas, A. A. Ethanol Production From Cassava Flour in Simultaneous Enzymatic Hydrolysis and Fermentation System, pp. 158-166. 2011.

28. Dagnino, E. P; Chamorro, E. R; Romano, S. D; Felissia, F. E; Area, M. C. Optimization of the acid pretreatment of rice hulls to obtain fermentable sugars for bioethanol production. Ind. Crops Prod. pp. 363-368.2013.

29. Vallejos, M. E; Kruyeniski, J; Area, M. C. Second-generation bioethanol from industrial wood waste of South American species. Biofuel Res. J. pp. 654-667.2017.

30. Romaní, A ; Ruiz, H. A; Pereira F. B;Teixeira, J. A ; Domingues, L. Integrated approach for effective bioethanol production using whole slurry from autohydrolyzed Eucalyptus globulus wood at high-solid loadings. Pp. 482-491. 2014.

31. Wood, I. P. y col. Comparison of saccharification and fermentation of steam exploded rice straw and rice husk. Biotechnol. Biofuels. pp. 1-9. 2016.

32. Singh, A.; Bajar, S; Bishnoi, N. R. Enzymatic hydrolysis of microwave alkali pretreated rice husk for ethanol production by Saccharomyces cerevisiae, Scheffersomyces stipitis and their co-culture. Fuel. pp. 699-702. 2014. 
33. Ye Chen, H. X ; Stevens, M. A ; Zhu, Y. Understanding of alkaline pretreatment parameters for corn stover enzymatic saccharification. Biotechnol. Biofuels. pp. 2-10. 2013.

Recibido: 25/02/2019.

Aprobado: 01/08/2019. 